\title{
Nonradial Modes in RR Lyrae Stars
}

\author{
W. A. Dziembowski \\ Warsaw University Observatory, Al. Ujazdowskie 4, 00-478 Warszawa, \\ Poland
}

S. Cassisi

Osservatorio Astronomico Collurania, I-64100, Teramo, Italy

\begin{abstract}
We surveyed nonradial mode properties in evolutionary sequences of RR Lyrae star models, focusing attention on modes that may be driven by the opacity mechanism and on those that may be excited as a consequence of the 1:1 resonance with the radial pulsation. Our survey shows that all RR Lyrae star models share all qualitative properties of the nonradial modes. There is always a large number of unstable low degree modes with frequencies close to unstable radial modes. However, owing to higher mode inertia, for most of nonradial modes the driving rates are much lower than those for radial modes. The exceptions are the strongly trapped (STU) modes which begin with $\ell$ degrees 7 to 10 (depending on the model) at frequencies somewhat above the fundamental radial mode, and with $\ell=5$ or 6 at frequencies somewhat above the radial mode overtones. We argued that these modes are not likely candidates for identification of oscillations recently detected in some RR Lyrae stars. More likely candidates seem, in spite of their lower driving rates, the $\ell=1$ modes.

We found also that parameters which determine the chances of the excitation of nonradial modes through the 1:1 resonance do not vary much over the range of RR Lyrae star parameters. According to our estimate the excitation has a high probability. In fact, some nonradial modes should be excited in the majority of the RRab pulsators and in a significant fraction of $(\sim 30 \%)$ of RRc pulsators. The actual number is likely greater because we ignored the effect of rotation, which increases the probability of resonant excitation. The instability may lead to a periodic light curve modulation, that is to the Blazkho effect, if the nonlinear interaction results in a periodic energy exchange between the radial and the nonradial modes. A light curve modulation may also take place in the case of a constant amplitude limit cycle if the nonradial mode is of low degree and it is not symmetric about the rotation axis.
\end{abstract}

See Dziembowski \& Cassisi (1999) for the full paper.

\section{Reference}

Dziembowski, W. A. \& Cassisi, S. 1999, Acta Astron., 49, 371 

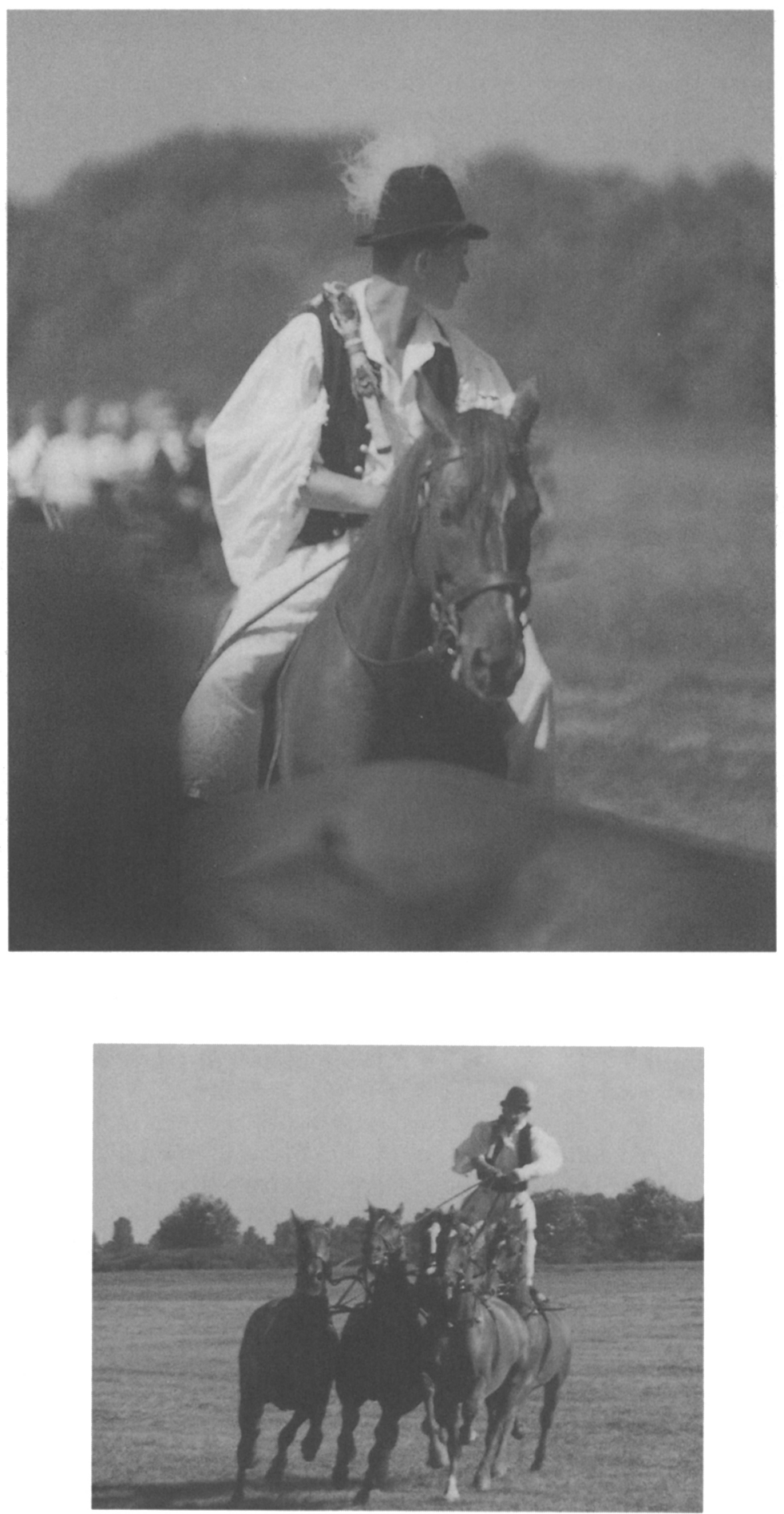